Methods 74 patients (93 stents) with OCT post-stent implantation were included in the study. Cross-sectional OCT images were analysed at $1-\mathrm{mm}$ intervals (every 15 frames), and 302 crosssectional images with lipid or calcific lesions under the stent struts were selected. The struts in these sections were divided into four groups according to the plaque conditions under the struts: group 1 -struts on the normal vessel, group 2-on the fibrotic lesion, group 3-on the calcific lesion, group 4-on the lipid rich lesion. The neointimal hyperplasia thickness on the stent struts were measured by OCT.

Results The plaque conditions under 806 struts could be detected clearly by OCT. Among them, 157 struts were on the normal vascular wall (the intimal thickness were less than $250 \mu \mathrm{m}$ by OCT), 344 struts were on the fibrotic lesions, 145 struts on calcific lesions and 160 struts on lipid lesions. The neointimal thickness were $0.132 \pm 0.081 \mu \mathrm{m}, \quad 0.148 \pm 0.091 \mu \mathrm{m}, \quad 0.150 \pm 0.105 \mu \mathrm{m}$ and $0.166 \pm 0.088 \mu \mathrm{m}$ respectively in group $1-4$. The $\mathrm{p}$ value was 0.011 . Conclusions The plaque type has impact on the in-stent neointimal hyperplasia. The underlying lipid or calcific components in plaque may promote neointimal hyperplasia after stent implantation.

\section{E0321 THE CORRELATION OF CARDIAC TROPONIN I AND DECOMPENSATED HEART FAILURE}

doi:10.1136/hrt.2010.208967.321

Zhaoxiufen Sunxiaofei, Zhaoxiufen Sunxiaofei. The First People's Hospital, Shan Dong Ji Ning

Objective Correlation of Cardiac Troponin I and Decompensated heart failure. Small studies report that $\mathrm{cTnI}$ is elevated in severe heart failure (HF) and may predict adverse outcomes.

Methods Serial blood samples were instantly collected from 336 patients who presented Decompensated heart failure (NYHA class III-IV) at Admission. Patients with acute myocardial infarction or myocarditis were excluded from analysis. Measurement of cTnI and Brain natriuretic peptide (BNP), echocardiography was done after Disease condition Improved. cTnI was detectable (cTnI $\geq 0.40 \mathrm{ng} /$ $\mathrm{ml}$ ) in serum of 161 patients (47.9\%). Patients with detectable cTnI levels had significantly higher B-type natriuretic peptide (BNP) levels $(p<0.001)$. A significant correlation was found between detectable cTnI and progressive decline in ejection fraction over time. Furthermore, detectable cTnI was associated with increased mortality risk (RR, 2.57; 95\% CI, 1.43 to 3.78). After adjustment of other factors associated with adverse prognosis including age, sex, ejection fraction, and coronary artery disease, cTnI remained a significant predictor of death. cTnI used in conjunction with BNP further improved prognostic value.

Conclusions cTnI is associated with elevated BNP levels and progressive left ventricular dysfunction in patients with severe HF. cTnI may be a novel, useful tool in identifying patients with HF who are at increased risk for progressive ventricular dysfunction and death

\section{e0322 TO INVESTIGATE INAPPROPRIATE DISCHARGE OF}

doi:10.1136/hrt.2010.208967.322

Xinxia Zhang, Xuesong Hu, Xiangguang Xu, Weihua Fang, Changlong Pen. Department of Cardiology, Shenzhen Futian Hospital

Objective In order to avoid and decrease inappropriate discharge caused by implantable cardioverter defibrillators (ICD), we analysed the cause of inappropriate discharge.

Methods Clinical follow-up data of inappropriate discharges by ICD in 6 patients were studied. All the 6 patients were male, 2 were dual chamber ICD, while 7 were single chamber ICD. The patients were followed-up from 0.5 to 9 (averaged 4.5) years.

Results During follow-up, all the patients survived. Arrhythmias were detected 358 times, ICD treatment was performed 71 times, inappropriate discharges occurred 14 times. 2 patients who implanted ICD earlier experienced all the 14 inappropriate discharges. The causes of inappropriate discharges include oversensing sinus tachycardia (3 times), electromagnetic interference (2 times), lead insulation breaks (9 times). They accounted for $21.4 \%, 14.2 \%, 64.2 \%$ of total inappropriate discharges, respectively. Conclusion Inappropriate discharge of ICD should be taken seriously consideration.

\section{e0323 ANALYSIS OF ADVERSE EFFECTS FROM STATINS-BASED MULTI-DRUG MEDICATION}

doi:10.1136/hrt.2010.208967.323

Zhao Mei, Zhao Zhengdang, Shi Jin, Chen Yanli, Li Hong, Zou Deling, Ma Shumei, Li Xiaodong. Deptartment of Cardiology, Shengjing Hospital of China Medical University

Objective To investigate risk factors and possible mechanisms of adverse effects from statins-based multi-drug medication.

Methods The medical materials from eleven patients who suffered from adverse effects of statins-based multi-drug medication were collected and analysed.

Results 7 patients who took simvastatin $40 \mathrm{mg} /$ day for monotherapy for four weeks produced normal laboratory tests results. Four patients who took a combination of ezetimibe/simvastatin 10/ $40 \mathrm{mg} /$ day for 4 weeks, 4 patients who took a combination of simvastatin $40 \mathrm{mg} /$ day plus niacin for 8 weeks and 3 patients who took a combination of ezetimibe/simvastatin $10 / 40 \mathrm{mg} /$ day plus niacin for eight weeks or less showed hepatic dysfunction (Alt $>1.5 \mathrm{ULN})$ and/or amyasthenia (CK $>1.5 \mathrm{ULN})$ and finally discontinued medication

Conclusion For females, age, polypharmacy and drug interactions are very important risk factors for statins-based multi-drug medication. One of the possible mechanisms is that other interacting drugs competitively inhibit the common pathway by which the drugs are metabolised, resulting in increased statins exposure, subsequent hepatotoxicity and skeletal muscle toxicity.

\section{e0324 PLATELET ACTIVATION DISTRIBUTION IN CHINESE HIGH RISK PATIENTS WITH ACUTE CORONARY SYNDROME}

doi:10.1136/hrt.2010.208967.324

${ }^{1}$ Ren Yihong, 'Chen Yundai, ${ }^{1}$ Zhao Ming, ${ }^{2}$ Chen Jinsong, ${ }^{1}$ Chen Lian, ${ }^{1}$ Liu Hongbin, ${ }^{1}$ Wang Yu, ${ }^{1}$ Snu Zhijun. ${ }^{1}$ PLA General Hospital/Cardiovascular Department; ${ }^{2}$ PLA General Hospital, Clinical Laboratory

Background All cases of ACS treated with the same doses antiplatelet remedy without considering their results from it is not an optimal remedy obviously. TEG-Mapping assay can offer us a possibility to detect the activity of platelet in general clinical therapy process.

Objectives To evaluate the antiplatelet therapy results immediately post- PCI in high risk ACS patients.

Methods All ACS patients $(n=310)$ with elective PCI took aspirin loading dose $300 \mathrm{mg}$ before PCI and followed by $100 \mathrm{mg} /$ day and clopidogrel loading dose $600 \mathrm{mg}$ before PCI and followed by $75 \mathrm{mg} /$ day therapy. Potential and residual platelet activity in ADP and AA pathway were detected respectively after $24-48$ h from PCI with modified TEG-mapping assay. 